\title{
Single-port thoracoscopic anatomic resection for chronic inflammatory lung disease
}

\author{
Chen-Yu Wu ${ }^{1}$, Ying-Yuan Chen ${ }^{1,2}$, Chao-Chun Chang ${ }^{1}$, Yi-Ting Yen ${ }^{1}$, Wu-Wei Lai ${ }^{1}$, Wei-Li Huang ${ }^{1,2^{*}}$ and \\ Yau-Lin Tseng ${ }^{1}$
}

\begin{abstract}
Background: It is challenging to proceed thoracoscopic anatomic resection when encountering severe pleural adhesion or calcified peribronchial lymphadenopathy. Compared with multiple-port video-assisted thoracoscopic surgery (MP-VATS), how to overcome these challenges in single-port (SP-) VATS is still an intractable problem. In the present study, we reported the surgical results of chronic inflammatory lung disease and shared some useful SP-VATS techniques.
\end{abstract}

Methods: We retrospectively assessed the surgical results of chronic inflammatory lung disease, primarily bronchiectasis, and mycobacterial infection, at our institution between 2010 and 2018. The patients who underwent SP-VATS anatomic resection were compared with those who underwent MP-VATS procedures. We analyzed the baseline characteristics, perioperative data, and postoperative outcomes, and illustrated four special techniques depending on the situation: flexible hook electrocautery, hilum-first technique, application of Satinsky vascular clamp, and staged closure of bronchial stump method.

Results: We classified 170 consecutive patients undergoing thoracoscopic anatomic resection into SP and MP groups, which had significant between-group differences in operation time and overall complication rate $(P=0.037$ and 0.018 , respectively). Compared to the MP-VATS group, the operation time of SP-VATS was shorter, and the conversion rate of SP-VATS was relatively lower $(3.1 \%$ vs. $10.5 \%, P=0.135)$. The most common complication was prolonged air leakage (SP-VATS, 10.8\%; MP-VATS, 2.9\%, $P=0.045$ ).

Conclusions: For chronic inflammatory lung disease, certain surgical techniques render SP-VATS anatomic resection feasible and safe with a lower conversion rate.

Keywords: Anatomic resection, Bronchiectasis, Inflammatory lung disease, Mycobacterial infection, Single-port, Video-assisted thoracoscopic surgery

\section{Introduction}

Minimally invasive surgery has become known worldwide to treat cancer because of the advantages of smaller surgical wounds, such as less acute pain and chronic paresthesia [1, 2]. However, in cases of long-term lung

\footnotetext{
*Correspondence: enozic@hotmail.com

${ }^{1}$ Division of Thoracic Surgery, Department of Surgery, National Cheng Kung University Hospital, College of Medicine, National Cheng Chung University, 138 Sheng-Li Road, Tainan 704, Taiwan

Full list of author information is available at the end of the article
}

inflammation, severe hypervascularized pleural adhesion and calcified peribronchial lymph nodes (LNs) develop, making it challenging to proceed with thoracoscopic anatomic resection. Therefore, many surgeons prefer thoracotomy than minimally invasive surgery for chronic inflammatory lung diseases [3].

With the advancement of instruments and surgical techniques, video-assisted thoracoscopic surgery (VATS) is also used to treat symptomatic inflammatory lung disease, improving quality of life. The use of multiple-port 
(MP-) VATS for bronchiectasis, aspergillosis, and tuberculosis, has been reported previously [3-5]. We also published our results, showing the feasibility of MP-VATS lung resection for pulmonary tuberculosis [6-8]. Furthermore, we reported the outcome of single-port (SP-) VATS on pulmonary tuberculosis and sequestration despite the small number of cases $[8,9]$. Compared to MP-VATS, the working space for instruments is so limited in SP-VATS, impeding the management of pleural adhesion, fissure symphysis, and calcified peribronchial LNs. Therefore, some special techniques should be applied to prevent unexpected thoracotomy. With more experiences in the past years, we had developed several useful techniques to overcome these difficulties. In the current study focused on chronic inflammatory lung disease, primarily bronchiectasis and mycobacterial infection, we shared the surgical outcomes of SP-VATS anatomic lung resection, compared to MP-VATS, and emphasized the importance of these techniques in preventing thoracotomy conversion and intraoperative complications.

\section{Patients and methods}

\section{Patients and data collection}

We reviewed all patients who underwent VATS anatomic resection for pulmonary mycobacterial infection or symptomatic bronchiectasis between January 2010 and December 2018 at the National Cheng Kung University Hospital using data from the database of thoracic surgery and medical records. If the chronic lung disease is characterized by chronic cough, recurrent pulmonary infection, life-threatening hemoptysis, and localized lung parenchyma change on computed tomography, we advise surgical resection to control infection and improve the quality of life. Besides, some patients of mycobacterial infection were enrolled as surgical candidates when cavitated lesions or destructed lung become bacterial refuges. Over the last few years of study period, SP-VATS has replaced MP-VATS and become the standard manner of surgical approach. However, when the preoperative computed tomography revealed the central hilar area is significantly involved by these lesions, we absolutely choose the intentional open thoracotomy because handassisted hemostasis is usually required in these situations. We excluded those with simultaneous lung abscess and empyema because of acute infection entity. This study, which did not require an informed consent, was approved by the National Cheng Kung University Hospital Institutional Review Board (approval no. B-ER-107-108).

We classified patients into two groups: SP-VATS and MP-VATS. The statistical analysis includes the following variables: (1) demographic and clinical information (i.e., sex, age, disease etiology, comorbidity, preoperative pulmonary function test, surgical indications, sidedness, extent of resection, and procedure type) and (2) the operation characteristics (i.e., the presence of calcified LNs, adhesion score, operation time, major vessel injury, the presence of conversion, chest tube duration, intensive care unit and hospital stay, and complications).

\section{Calcified LN and total adhesion score}

Calcified LN is the calcification of LNs surrounding the bronchovascular bundle of the target lobe by the mediastinal window of the preoperative chest computed tomography ( $40 \mathrm{H}$ level and $300 \mathrm{H}$ width). When calcified LNs are present, the MP-VATS approach may be chosen intentionally depending on the surgeon's preference.

The total adhesion score is the sum of the pleural adhesion score and fissure adhesion scores. The first intraoperative step was determining the individual severity of pleural and fissure adhesions subjectively as follows: 0 represents no or only focal adhesion; 1 limited adhesion around the target area with less than $50 \%$ of pleural cavity or interlobar area; and 2 diffuse adhesion or fused fissure with more than $50 \%$ of pleural cavity or interlobar area. Upon VATS exploration, we evaluated SP-VATS' feasibility using the total adhesion score. If the score is $\geqq 3$ points, the surgeon could change to MP-VATS based on his judgement.

\section{Surgical procedure}

All patients were intubated with a double-lumen endotracheal tube by an experienced anesthesiologist and placed in the lateral decubitus position. A single incision, measuring approximately $4 \mathrm{~cm}$ in length, was made in the fourth or fifth intercostal space at the anterior axillary line. The surgical techniques and instruments used in SP-VATS were similar to those used in MP-VATS. If unexpected situations during VATS require conversion, minithoracotomy will be performed. Typically, we divided the pulmonary arteries first to avoid congestion of the targeted lobe. The pulmonary veins and the bronchus were then dissected separately. The bronchus was divided using a linear stapler (Echelon Flex Endocutter, Johnson \& Johnson, USA), but bronchial stump reinforcement was not routinely performed. After meticulous air leakage control, we placed a $24 \mathrm{Fr}$ chest tube.

\section{Special techniques}

Because the limitations of SP-VATS, such as being a single-direction approach and using few instruments in the utility wound, we developed certain useful techniques to facilitate anatomic resection (Fig. 1).

First is the flexible hook electrocautery (Fig. 1A). Generally, blunt dissection using fingers or small peanut gauzes managed dense pleural adhesions. L-hook electrocautery and energy-based instruments were used to 


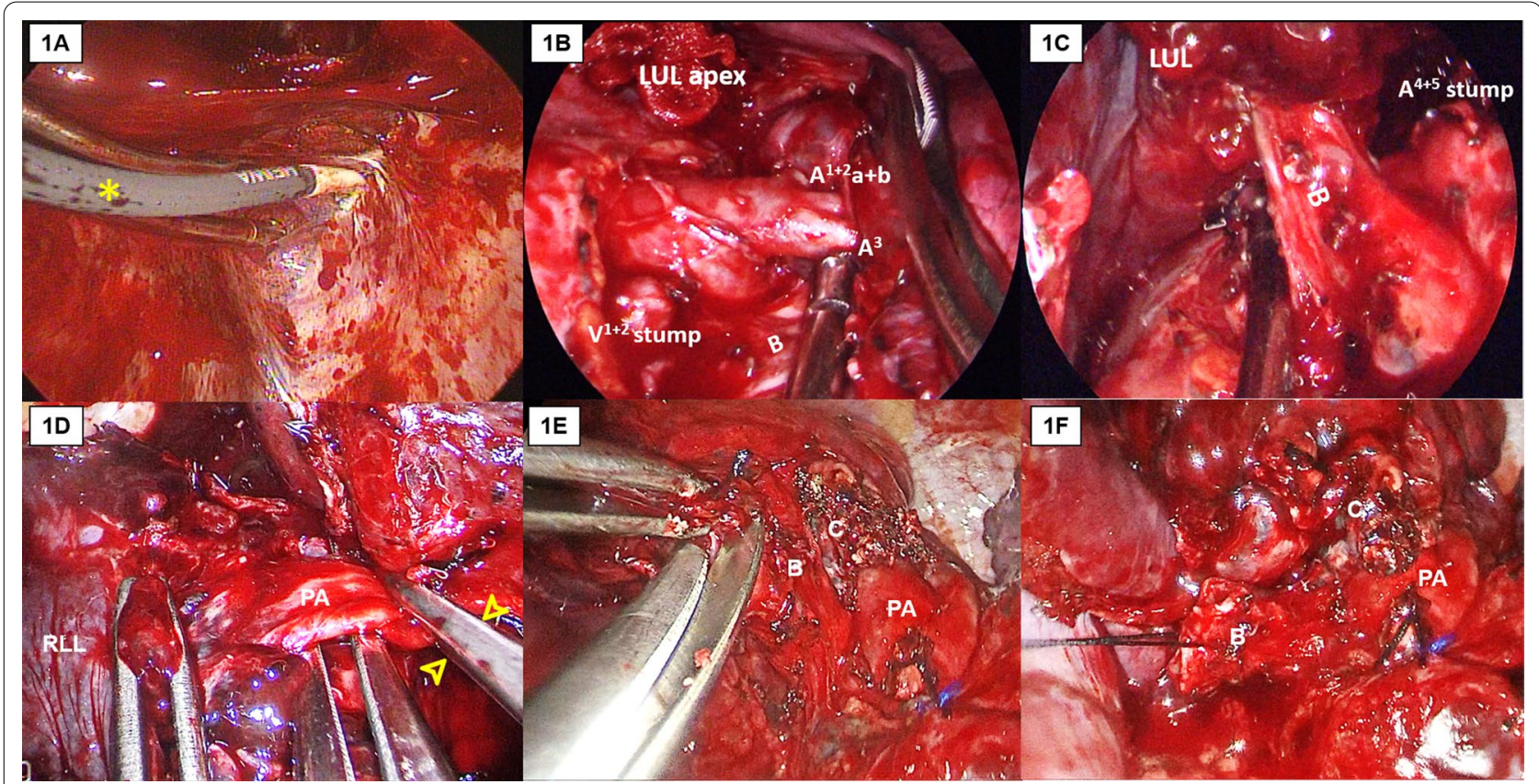

Fig. 1 Special techniques for SP-VATS anatomic resection in chronic inflammatory lung disease. The first method is the use of a flexible hook electrocautery for apical or costophrenic adhesion (A, asterisk). The second method is the hilum-first technique, which can be used when the hilum area is relatively less adhesive than the apical area in mycobacterial infection. The LUL apex was still adhesive to the chest wall during hilar dissection (B). All vascular branches to the LUL were divided, followed by LUL bronchus transection (C). The last step was the lysis of the apical adhesion. The third method is the use of a Satinsky vascular clamp for bleeding prevention or control ( $\mathbf{D}$, arrows). The fourth method is bronchial stump staged closure when calcified LNs hinder the separation of the bronchus and vascular structure (E and F). B bronchus, C calcified lymph node, LUL left upper lobe, PA pulmonary artery, RLL right lower lobe

accelerate adhesiolysis. However, it is hard to use linear instruments for pleural adhesion in the apical or costophrenic area. So, we used flexible hook electrocautery to complete adhesiolysis.

The second technique is the hilum-first approach. After VATS exploration and adhesion severity evaluation, we could begin with the dissection of vascular structure if hilar adhesion was relatively minor rather than a dense pleural adhesion (Fig. 1B, C). Pleural adhesion could be used as counter traction, and we manipulate a single energy device and a suction device to avoid instrument fencing.

The third technique is the use of Satinsky vascular clamp (Fig. 1D). When faced with the occurrence of major bleeding or fear of main pulmonary artery injury during dissection, we apply a Satinsky vascular clamp immediately via the utility wound to clamp the vessel and bronchus concomitantly to control or prevent bleeding.

The final technique is the staged closure of the bronchial stump method (Fig. 1E). We applied this technique when segmental pulmonary artery is behind the bronchus and unable to be skeletonized due to calcified LNs. Using endoscopic scissors, the bronchus can be transected and the fibrocalcified tissue can be dissected sequentially along the posterior wall of the opened bronchial stump step by step. Then, the segmental pulmonary arteries can be looped and divided sequentially using either suture ligation or stapler device. Finally, the opened bronchial stump can be lifted by stay sutures and closed using a linear stapler (Fig. 1F).

\section{Statistical analysis}

Descriptive statistics were used to assess the patients' demographic and perioperative characteristics. Continuous data were expressed as means \pm standard deviation, whereas categorical data were expressed as frequencies and proportions. Continuous variables were analyzed using the Mann-Whitney test, and categorical variables using the chi-squared test. A probability value of less than 0.05 was considered statistically significant. All statistical analyses were performed using IBM SPSS Statistics for Windows, version 19. (IBM Corp., Armonk, N.Y).

\section{Results}

Between January 2010 and December 2018, 181 patients underwent anatomic resection for bronchiectasis or pulmonary mycobacterial infection in our hospital. We performed VATS intentionally for 170 patients and open 
thoracotomy for 11 patients. Figure 2 shows the distribution of surgical approach. We began using SP-VATS in 2015 and generally adapted this method for lung surgery rapidly. By 2018, we used SP-VATS for approximately $87 \%$ of patients with chronic inflammatory lung disease. Only three patients underwent MP-VATS, one of whom had severe pleural and fissure adhesion (adhesion score 4) and two based on the surgeon's preference (both adhesion score 0 ).

Table 1 lists the characteristics of patients who underwent VATS anatomic resection, with no significant between-group difference on age, sex, etiology, pulmonary function test, and surgical indication. The percentage of pulmonary mycobacterial infection in MP-VATS was slightly higher than that in SP-VATS $(60 \%$ vs. $52.3 \%$; $P=0.325)$. A total of $72(42.4 \%)$ patients had comorbidities, including $21(32.3 \%)$ who underwent SP-VATS and 51 (48.6\%) MP-VATS. The prevalence of diabetes mellitus was significantly higher in the MP-VATS group than in the SP-VATS (32.4\% vs. $18.5 \%$; $P=0.047$ ).

The sidedness and extent of resection, as well as surgical procedure, were also similar between the two groups (Table 1). A total of 181 anatomic resection procedures were performed, including 88 lobectomies and $93 \mathrm{seg}$ mentectomies. Among these patients, four underwent simultaneous bilateral anatomic resection (three for bronchiectasis and one for nontuberculous mycobacterial infection).

Table 2 summarizes the surgical characteristics of the two groups. No significant between-group difference was observed on calcified LN, the severity of pleural / fissure adhesion, and adhesion score. Although the percentage of high adhesion score $($ score $=4$ ) in the MP-VATS group was higher than that in the SP-VATS group, the comparison was not statistically significant (score $0-3$ vs. score $4 ; P=0.113$ ). No significant difference was also observed on intraoperative blood loss, major vessel injury, conversion rate, chest tube duration, intensive care unit stay, or postoperative hospital stay. SP-VATS patients had less operation time but a higher complication rate than MP-VATS patients $(P=0.037$ and 0.018 , respectively). Two (3.1\%) patients were converted to mini-thoracotomy for bleeding control, both of which were in the early period of SP-VATS development. There was no 30-day mortality in either group.

Table 3 lists the details regarding the complications. On the whole, $34(20 \%)$ patients experienced complications and $26(15.3 \%)$ had pulmonary-related complications. The most common complication was prolonged air leakage ( $>7$ days), followed by post-surgical empyema (5.9\% and $4.1 \%)$. The SP-VATS group had a significantly higher percentage of prolonged air leakage $(10.8 \%$ vs. $2.9 \%$, $P=0.045)$.

Among the 65 consecutive patients of SP-VATS, patients 1-33 constitute the first half phase and patient 34-65 constituted the second half phase. Table 4 demonstrated the complication rate regarding the different phase. No significant between-group differences were observed in each complication, but the overall

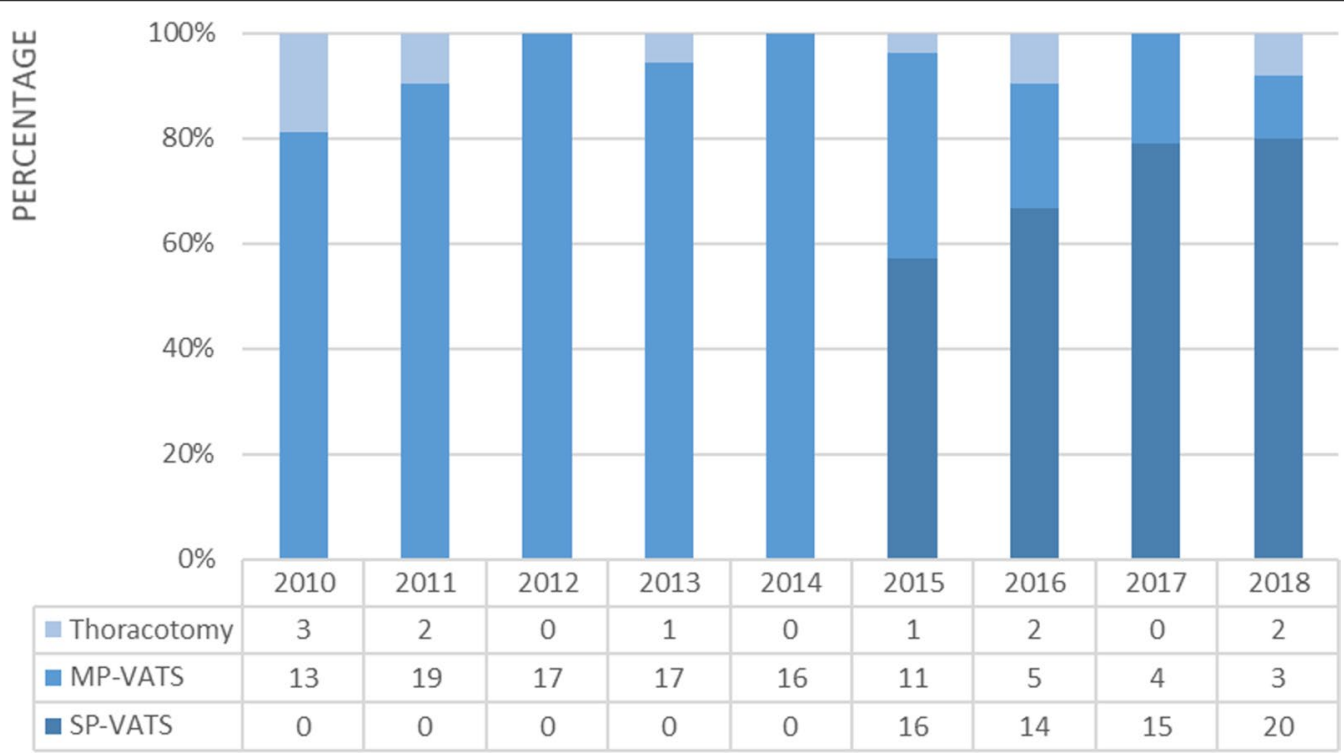

Fig. 2 VATS anatomic resection for chronic inflammatory lung disease, primarily bronchiectasis, and mycobacterial infection, at the National Cheng Kung University Hospital. The patients intentionally undergoing open thoracotomy are also listed 
Table 1 Patient characteristics by surgical approach

\begin{tabular}{|c|c|c|c|}
\hline & $\begin{array}{l}\text { SP-VATS } \\
(\mathrm{n}=65)\end{array}$ & $\begin{array}{l}\text { MP-VATS } \\
(n=105)\end{array}$ & $P$-value \\
\hline Age, mean $\pm S D$ & $54.2 \pm 12.6$ & $52.4 \pm 12.5$ & 0.351 \\
\hline Sex, male (\%) & $33(50.8)$ & $56(53.3)$ & 0.745 \\
\hline Etiology, n (\%) & & & 0.325 \\
\hline Bronchiectasis & $31(47.7)$ & $42(40.0)$ & \\
\hline TB/NTM infection & $34(52.3)$ & $63(60.0)$ & \\
\hline \multicolumn{4}{|l|}{ Comorbidity, n (\%) } \\
\hline Hypertension & $12(18.5)$ & $18(17.1)$ & 0.827 \\
\hline Diabetes mellitus & $12(18.5)$ & $34(32.4)$ & 0.047 \\
\hline Coronary artery disease & $1(1.5)$ & $3(2.9)$ & 1.000 \\
\hline GERD & 0 & $3(2.9)$ & 0.287 \\
\hline CKD/ESRD & $1(1.5)$ & $3(2.9)$ & 1.000 \\
\hline Liver cirrhosis & $1(1.5)$ & $3(2.9)$ & 1.000 \\
\hline Malignancy history & $3(4.6)$ & $2(1.9)$ & 0.372 \\
\hline \multicolumn{4}{|c|}{ Pulmonary function test, mean $\pm S D$} \\
\hline FEV1 (L) & $2.16 \pm 0.84$ & $2.27 \pm 0.80$ & 0.458 \\
\hline PEF1/FVC (\%) & $76.9 \pm 11.0$ & $77.5 \pm 10.3$ & 0.755 \\
\hline Surgical indication, n (\%) & & & 0.066 \\
\hline Hemoptysis & $36(55.4)$ & $48(45.7)$ & \\
\hline Recurrent pneumonia & $11(16.9)$ & $16(15.2)$ & \\
\hline Cavity, infected & $11(16.9)$ & $13(12.4)$ & \\
\hline Destroyed lobe & $2(3.1)$ & $4(3.8)$ & \\
\hline TB medication failure & $3(4.6)$ & $23(21.9)$ & \\
\hline Undetermined mass & $2(3.1)$ & $1(1.0)$ & \\
\hline Sidedness, n (\%) & & & 0.281 \\
\hline Right & $35(53.8)$ & $55(52.4)$ & \\
\hline Left & $27(41.5)$ & $49(46.7)$ & \\
\hline Bilateral & $3(4.6)$ & $2(1.0)$ & \\
\hline Extent of resection, n (\%) & & & 0.435 \\
\hline Upper lobe & $32(49.2)$ & $63(60.0)$ & \\
\hline Middle lobe & $9(13.8)$ & $16(15.2)$ & \\
\hline Lower lobe & $15(23.1)$ & $20(19.0)$ & \\
\hline Upper + middle lobe & $3(4.6)$ & $2(1.9)$ & \\
\hline Upper+ lower lobe & $3(4.6)$ & $3(2.9)$ & \\
\hline Middle + lower lobe & $3(4.6)$ & $1(1.0)$ & \\
\hline Surgical procedure, n (\%) & & & 0.226 \\
\hline Lobectomy & $27(41.5)$ & $52(49.5)$ & \\
\hline Segmentectomy & $28(43.1)$ & $47(44.8)$ & \\
\hline Lob.+ Seg., ipsilateral & $6(9.2)$ & $4(3.8)$ & \\
\hline Lob. + Seg., bilateral & $1(1.5)$ & $1(1.0)$ & \\
\hline Seg.+Seg., ipsilateral & 0 & $1(1.0)$ & \\
\hline Seg.+ Seg., bilateral & $2(3.1)$ & 0 & \\
\hline Bilobectomy & $1(1.5)$ & 0 & \\
\hline
\end{tabular}

CKD/ESRD chronic kidney disease/end-stage renal disease, FEV1 forced expiratory volume in $1 \mathrm{~s}, F V C$ forced vital capacity, GERD gastroesophageal reflux disease, Lob. Lobectomy, NTM nontuberculous mycobacteria, SD standard deviation, Seg. Segmentectomy, TB tuberculosis complication rate showed a decreasing trend in the second half phase.

\section{Comment}

Dense pleural adhesion is a relative contraindication of VATS [4]. With advancements in thoracoscopic video systems and surgical techniques, VATS anatomic resection can be applied to chronic inflammatory lungs even with pleural adhesions, including pulmonary mycobacterial infection, bronchiectasis, or lung cancer after neoadjuvant treatment. Currently, pleural or fissure adhesions do not preclude proceeding with MP-VATS [10]. However, literature focused on how to overcome dense adhesion using SP-VATS is still limited. We reported our experiences on dealing with pulmonary tuberculosis by thoracotomy and MP-VATS previously [9]. With welldeveloped VATS skills, we began performing SP-VATS anatomic resection in 2015, the results of which were reported in the current study. On the basis of these experiences, we established several strategies to overcome some of this technique's difficulties.

First is using appropriate instrument for pleural adhesiolysis. In SP-VATS, it is relatively easy to release mediastinal and mid-thoracic pleural adhesion by electrocautery. However, for the adhesion of the lung apex, particularly the anterior apical area, bended electrode, or straight L-hook electrocautery could barely reach it. To overcome this limitation, a flexible L-hook electrocautery is used to release pleural adhesion in these tricky areas. Mun et al. also described a similar experience with the management of pleural adhesion via three- or four-port VATS [11]. Using suitable instruments for adhesiolysis, lung parenchyma injury, which leads to intraoperative bleeding or postoperative air leakage, can also be decreased.

Second is the management strategies of pleural and fissure adhesion. In SP-VATS, the most important is the single-direction manner, either anterior-to-posterior or caudal-to-cranial approach [12]. In our experience, around one-fourth of the patients with chronic inflammatory disease wound have severely adhesive or fused fissure, which resulted in higher risk of vessel injury during fissure opening. Fissure-last single-direction approach is better than fissure-first approach in decreasing the difficulty of vascular skeletonization and reducing the possibility of conversion and also decreasing the incidence of postoperative prolonged air leakage as the fissure is divided at the last step using staplers [13].

As facing apical adhesion usually caused by tuberculosis, the hilum-first technique, which we called apical adhesion-last approach, is an effective method. We had observed that some patients with chronic inflammatory lung disease, especially pulmonary tuberculosis, have intense apical pleural adhesion but relatively minor hilar 
Table 2 Perioperative data of SP- and MP-VATS anatomic resection for chronic inflammatory lung disease

\begin{tabular}{|c|c|c|c|}
\hline & $\begin{array}{l}\text { SP-VATS } \\
(n=65)\end{array}$ & $\begin{array}{l}\text { MP-VATS } \\
(n=105)\end{array}$ & $P$-value \\
\hline Calcified LNs, n (\%) & $8(12.3)$ & $20(19.0)$ & 0.250 \\
\hline Pleural adhesion (A), n (\%) & & & 0.269 \\
\hline No or focal (0) & $44(67.7)$ & $58(55.2)$ & \\
\hline Limited, $<50 \%$ of pleural cavity ( 1 ) & $9(13.8)$ & $19(18.1)$ & \\
\hline Diffuse, $\geqq 50 \%$ of pleural cavity ( 2 ) & $12(18.5)$ & $28(26.7)$ & \\
\hline Fissure adhesion (B), n (\%) & & & 0.226 \\
\hline No or focal $(0)$ & $33(50.8)$ & $57(54.3)$ & \\
\hline Limited, $<50 \%$ of interlobar area (1) & $18(27.7)$ & $18(17.1)$ & \\
\hline Diffuse or fused, $\geqq 50 \%$ of interlobar area (2) & $14(21.5)$ & $30(28.6)$ & \\
\hline Adhesion score $(A+B), n(\%)$ & & & 0.221 \\
\hline 0 & $24(36.9)$ & $43(41.0)$ & \\
\hline 1 & $20(30.8)$ & $18(17.1)$ & \\
\hline 2 & $9(13.8)$ & $14(13.3)$ & \\
\hline 3 & $6(9.2)$ & $11(10.5)$ & \\
\hline 4 & $6(9.2)$ & $19(18.1)$ & \\
\hline Adhesion score, binary grading, $\mathrm{n}(\%)$ & & & 0.113 \\
\hline Low (Score 0-3) & $59(90.8)$ & $86(81.9)$ & \\
\hline High (Score 4) & $6(9.2)$ & $19(18.1)$ & \\
\hline Operation time $(\min )$, mean $\pm S D$ & $186.3 \pm 67.8$ & $211.6 \pm 79.7$ & 0.037 \\
\hline Intraoperative blood loss $(\mathrm{mL})$, mean \pm SD & $256.2 \pm 412.1$ & $300.0 \pm 431.4$ & 0.515 \\
\hline Major vessel injury, n (\%) ${ }^{\mathrm{a}}$ & $9(13.8)$ & $6(5.8)$ & 0.076 \\
\hline Conversion, n (\%) & $2(3.1)$ & $11(10.5)$ & 0.135 \\
\hline Chest tube duration (days) & & & 0.184 \\
\hline Mean $\pm S D$ & $6.7 \pm 15.4$ & $4.1 \pm 3.4$ & \\
\hline Median (IQR) & $3.0(3.3)$ & $3.0(1.0)$ & \\
\hline ICU stay (days) & & & 0.407 \\
\hline Mean $\pm S D$ & $0.7 \pm 1.6$ & $0.6 \pm 1.2$ & \\
\hline Median (IQR) & $0(1.0)$ & $0(1.0)$ & \\
\hline Postoperative hospital stay (days) & & & 0.107 \\
\hline Mean $\pm S D$ & $8.4 \pm 10.5$ & $6.2 \pm 3.6$ & \\
\hline Median (IQR) & $5.0(4.0)$ & $5.0(3.0)$ & \\
\hline Complication, n (\%) & $19(29.2)$ & $15(14.3)$ & 0.018 \\
\hline
\end{tabular}

ICU intensive care unit

${ }^{\text {a }}$ Major vessel injury is the injury of pulmonary artery, and suture repair is needed

adhesion. Dissecting this adhesion as the last step has several advantages. On the one hand, after transection of lobar arteries is done, oozing from lung parenchyma will decrease during pleura adhesiolysis. By contrast, it is difficult to control oozing from either neovascularized pleura or lung parenchyma if pleura adhesiolysis is done first. On the other hand, being separated from the chest wall, the poorly compliant upper lobe may obscure the surgical field and make hilar dissection difficult. For SP-VATS, it is definitively important to use fewer instruments to do more work simultaneously, such as suction, dissection, and traction. The adhesion on the roof can provide counter traction when approaching the hilum structure. This method is specifically beneficial for left upper lobe with chronic inflammatory disease resection.

The third method is concomitant clamping of the bronchovascular structures with temporary use of a Satinsky vascular clamp, which is feasible for lower or middle lobe resection if calcified LNs severely anchored to both the bronchus and lobar arteries. The simultaneous transection of the bronchus and the artery (simultaneously stapled lobectomy) has been reported [14-16]. When facing the fibrocalcified LN in bronchovascular buddle, however, we did not completely support this technique based on past experience of staple malformation and disruption, tissue tear, and massive bleeding from artery stump. 
Table 3 Complications of SP-VATS vS. MP-VATS anatomic resection for chronic inflammatory lung disease

\begin{tabular}{llll}
\hline & SP-VATS & MP-VATS & $P$-value \\
\hline Prolonged air leak> 7 days, n (\%) & $7(10.8)$ & $3(2.9)$ & 0.045 \\
Post-surgical empyema, n (\%) & $5(7.7)$ & $2(1.9)$ & 0.108 \\
Massive hemothorax, n (\%) & $1(1.5)$ & 0 & 0.382 \\
Massive hemoptysis, n (\%) & 0 & $2(1.9)$ & 0.525 \\
Pneumonia, n (\%) & $1(1.5)$ & $2(1.9)$ & 1.000 \\
Respiratory failure with tracheostomy, & $1(1.5)$ & 0 & 0.382 \\
$\quad$ n (\%) & & & \\
Wound infection, n (\%) & $1(1.5)$ & $2(1.9)$ & 1.000 \\
Arrhythmia, n (\%) & $2(3.1)$ & $3(2.9)$ & 1.000 \\
AUR, n (\%) & $1(1.5)$ & $1(1.0)$ & 1.000 \\
Others, $\mathrm{n}(\%)$ & $2(3.1)$ & 0 & 0.145
\end{tabular}

AUR acute urinary retention

${ }^{a}$ Others included one case of neutropenic fever and another of cerebrovascular accident

In addition, calcified LNs in the bronchovascular bundle also contribute to vascular injury when attempting to skeletonize vessels. Gonzalez-Rivas et al. shared their experience with bleeding control using SP-VATS [17]. However, we cannot apply these methods if there is not enough space between the bronchus and vessels. Using this technique, we seldom need to convert to open thoracotomy hastily when artery injury occurs but rather can repair the injured site calmly. Dividing all the tissues surrounding the bronchovascular bundles, which facilitate the application of the Satinsky clamp, is the point of this method.

The last method is staged closure of the bronchus, which we have described previously [18]. In contrast to the third method used for the lower and middle lobe, it is rather suitable for bilateral upper lobe resection. Some surgeons have also adapted this technique to overcome the adherence of calcified LNs to the bronchus and lobar arteries [19]. The key point is that the bronchus should be transected by scissors as distally as possible to preserve a longer stump, facilitating its closure with a linear stapler sequentially.

From MP- to SP-VATS approach, we have developed several useful techniques to facilitate VATS anatomic resection for chronic inflammatory lung disease $[9,18]$. Although the current study demonstrated that the incidence of intraoperative major vessel injury was still higher, the rate of conversion to thoracotomy was relatively lower in the SP-VATS group, and the betweengroup difference of intraoperative blood loss was minimal. This represents that we can manage these vascular injuries well by SP-VATS techniques. Noticeably, the operation time is significantly shorter in the SP-VATS group. We believed these are a result of the accumulation of VATS experience and proficiency of surgical techniques we provided. When it comes to postoperative recovery characteristics, because one patient with bronchiectasis had post-surgical empyema, resulting in prolonged chest tube drainage (116 days) and postoperative hospital stay (37 days), the average of chest tube drainage and postoperative hospital stay in SP-VATS is relatively higher than that in MP-VATS without statistical significance $(\mathrm{p}=0.184$ and 0.107 , respectively). Based on the results of the median and IQR values, we considered SPVATS to be a safe procedure as MP-VATS.

A higher complication rate with SP-VATS is unexpected, which is associated with insufficient experience in the developmental phase of SP-VATS. The primary complication was prolonged air leakage ( $>7$ days), which also resulted in a longer chest tube duration and longer

Table 4 The complication of SP-VATS anatomic resection for chronic inflammatory lung disease regarding the different phase

\begin{tabular}{|c|c|c|c|}
\hline & $\begin{array}{l}\text { First half phase } \\
n=33\end{array}$ & $\begin{array}{l}\text { Second half phase } \\
n=32\end{array}$ & $P$-value \\
\hline Overall, n (\%) & $12(36.4)$ & $7(21.9)$ & 0.202 \\
\hline Prolonged air leak > 7 days, n (\%) & $4(12.1)$ & $3(9.4)$ & 0.723 \\
\hline Post-surgical empyema, n (\%) & $3(9.1)$ & $2(6.2)$ & 0.670 \\
\hline Massive hemothorax, n (\%) & $1(3.0)$ & 0 & 0.325 \\
\hline Pneumonia, n (\%) & $1(3.0)$ & 0 & 0.325 \\
\hline Respiratory failure with tracheostomy, n (\%) & $1(3.0)$ & 0 & 0.325 \\
\hline Wound infection, n (\%) & 0 & $1(3.1)$ & 0.310 \\
\hline Arrhythmia, n (\%) & $1(3.0)$ & $1(3.1)$ & 0.982 \\
\hline AUR, n (\%) & $1(3.0)$ & 0 & 0.325 \\
\hline Others $^{\mathrm{a}}, \mathrm{n}(\%)$ & $2(6.1)$ & 0 & 0.160 \\
\hline
\end{tabular}

AUR acute urinary retention

${ }^{\text {a }}$ Others included one case of neutropenic fever and another of cerebrovascular accident 
hospital stay. The incidence of prolonged air leakage could be reduced when some prophylactic agents, such as fibrin glue or polyglycolic acid sheets (NEOVEIL), are applied, but they are not covered by our national health insurance [20]. Noticeably, there was no 30-day mortality in SP-VATS group although the complication rate is relatively higher.

This study had some limitations. First, this retrospective non-randomized study was conducted at a single institution, and the number of cases was small. Second, the severity of adhesion score is subjectively evaluated, and the long-term outcome data are limited. With increased experience in performing SP-VATS, the surgeon's tolerance and perseverance for managing adhesion and calcified lymph node gradually increase, but surgical approach planning still depends on surgeon's preference and disease complexity. In our view, minimally invasive surgery is a trend nowadays, and SP-VATS is widely discussed in lung cancer surgery. For chronic inflammatory lung disease, however, SP-VATS is still challenging. Therefore, we made a great effort to develop some techniques to facilitate SP-VATS anatomic resection. Moreover, these specifical techniques can also be applied in MP-VATS to reduce unexpected thoracotomy conversion.

In conclusion, pleural and fissure adhesion or calcified LNs around the vessels and bronchus are not obstacles for conducting SP-VATS. If the surgeon is acquainted with the techniques we have proposed, SP-VATS anatomic resection for chronic inflammatory lung disease could become more feasible and safer with a lower thoracotomy conversion rate.

\section{Acknowledgements}

We thank the Taiwan Centers for Disease Control for supporting this work. We are grateful to Dr. Sheng-Hsiang Lin and Ms. Chih-Hui Hsu for providing the statistical consulting services from the Biostatistics Consulting Center, Clinical Medicine Research Center, National Cheng Kung University Hospital.

\section{Authors' contributions}

WLH and YLT contributed to the research conception and design; WLH, YYC, YTY, WWL, and YLT contributed to the provision of study patients; CYW, WLH and CCC contributed the data collection and assembly; WLH, CCC, and YYC contributed to the data analysis and interpretation. All authors contributed to the writing review and editing. All authors read and approved the final manuscript.

\section{Funding}

No.

\section{Availability of data and materials}

The datasets used and/or analyzed during the current study are available from the corresponding author on reasonable request.

\section{Declarations}

Ethics approval and consent to participate

This study was approved by the institutional review board of the National Cheng Kung University Hospital (B-ER-107-108) performed in accordance with the Declaration of Helsinki. The Informed consent was waived by the NCKUH institutional review board.

\section{Consent for publication}

Not applicable.

\section{Competing interests}

The authors declare that they have no competing interests.

\section{Author details}

${ }^{1}$ Division of Thoracic Surgery, Department of Surgery, National Cheng Kung University Hospital, College of Medicine, National Cheng Chung University,

138 Sheng-Li Road, Tainan 704, Taiwan. ${ }^{2}$ Institute of Clinical Medicine, College of Medicine, National Cheng Kung University, Tainan, Taiwan.

Received: 28 January 2021 Accepted: 11 May 2021

Published online: 18 May 2021

\section{References}

1. Reinersman JM, Passera E, Rocco G. Overview of uniportal video-assisted thoracic surgery (VATS): past and present. Ann Cardiothorac Surg. 2016;5:112-7.

2. Yi E, Kim D, Kim K. Evolution of video-assisted thoracic surgery (VATS) techniques for lung cancer; minimizing surgical injury and expanding applications. Asian J Surg. 2016;39:264-6.

3. Baysungur V, Dogruyol T, Ocakcioglu I, Misirlioglu A, Evman S, Kanbur S, Alpay L, Tezel C. The feasibility of thoracoscopic resection in bronchiectasis. Surg Laparosc Endosc Percutan Tech. 2017:27:194-6.

4. Chen QK, Chen C, Chen XF, Jiang GN. Video-assisted thoracic surgery for pulmonary aspergilloma: a safe and effective procedure. Ann Thorac Surg. 2014;97:218-23

5. Han Y, Zhen D, Liu Z, Xu S, Liu S, Qin M, Zhou S, Yu D, Song X, Li Y, Xiao N, Su C, Shi K. Surgical treatment for pulmonary tuberculosis: is video-assisted thoracic surgery "better" than thoracotomy? J Thorac Dis. 2015;7:1452-8.

6. Yen YT, Wu MH, Lai WW, Chang JM, Hsu IL, Chen YY, Huang WL, Lee WC, Chang KW, Tseng YL. The role of video-assisted thoracoscopic surgery in therapeutic lung resection for pulmonary tuberculosis. Ann Thorac Surg. 2013;95:257-63.

7. Tseng YL, Chang JM, Liu YS, Cheng L, Chen YY, Wu MH, Lu CL, Yen YT. The role of video-assisted thoracoscopic therapeutic resection for medically failed pulmonary tuberculosis. Medicine (Baltimore). 2016;95:e3511.

8. Lin TH, Huang WL, Chang CC, Yen YT, Lai WW, Tseng YL, Chen YY. Uniportal video-assisted thoracoscopic surgery lobectomy and segmentectomy for pulmonary sequestration. J Thorac Dis. 2018;10:3722-8.

9. Tseng YL, Chang CC, Chen YY, Liu YS, Cheng L, Chang JM, Wu MH, Yen YT. From one incision to one port: the surgical technique and the evolution of segmentectomy in patients with pulmonary tuberculosis. PLoS ONE. 2018;13:e0197283.

10. Ocakcioglu I. Uniportal thoracoscopic treatment in bronchiectasis patients: preliminary experience. Wideochir Inne Tech Maloinwazyjne. 2019;14:304-10.

11. Mun M, Ichinose J, Matsuura Y, Nakao M, Okumura S. Tips and tricks for entering a difficult chest via VATS. JVis Surg. 2018;4:227.

12. Liu L, Che G, Pu Q, Ma L, Wu Y, Kan Q, Zhuge X, Shi L. A new concept of endoscopic lung cancer resection: single-direction thoracoscopic lobectomy. Surg Oncol. 2010;19:e71-77.

13. Stamenovic D, Bostanci K, Messerschmidt A, Jahn T, Schneider T. Fissureless fissure-last video-assisted thoracoscopic lobectomy for all lung lobes: a better alternative to decrease the incidence of prolonged air leak? Eur J Cardiothorac Surg. 2016;50:118-23.

14. Lewis RJ. Simultaneously stapled lobectomy: a safe technique for videoassisted thoracic surgery. J Thorac Cardiovasc Surg. 1995;109:619-25.

15. Qiang G, Nakajima J. Simultaneous stapling of pulmonary vein and bronchus in video-assisted thoracic surgery lobectomy. Ann Thorac Cardiovasc Surg. 2015;21:78-80.

16. Liu C, Ma L, Pu Q, Liao H, Liu L. How to deal with benign hilar or interlobar lymphadenopathy during video-assisted thoracoscopic surgery 
lobectomy-firing the bronchus and pulmonary artery together. J Vis Surg. 2016;2:26.

17. Gonzalez-Rivas D, StupnikT, Fernandez R, de la Torre M, Velasco C, Yang Y, Lee W, Jiang G. Intraoperative bleeding control by uniportal videoassisted thoracoscopic surgery †. Eur J Cardiothorac Surg. 2015;49:17-24.

18. Chen Y-Y, Lin T-H, Chang C-C, Huang W-L, Yen Y-T, Tseng Y-L. Staged bronchial closure in uniportal video-assisted thoracoscopic anatomical resection for lung cancer with calcified lymph nodes. J Vis Surg. 2017;3:149-149.

19. Lin F, Wei S, Liu C, Pu Q, Mei J, Liao H, Liu L. The technique of cutting open the bronchus during VATS left upper lobectomy with complicated hilar anatomy. J Thorac Dis. 2018;10:6269.
20. Kawai N, Kawaguchi T, Suzuki S, Yasukawa M, Tojo T, Taniguchi S. Lowvoltage coagulation, polyglycolic acid sheets, and fibrin glue to control air leaks in lung surgery. Gen Thorac Cardiovasc Surg. 2017;65:705-9.

\section{Publisher's Note}

Springer Nature remains neutral with regard to jurisdictional claims in published maps and institutional affiliations.
Ready to submit your research? Choose BMC and benefit from:

- fast, convenient online submission

- thorough peer review by experienced researchers in your field

- rapid publication on acceptance

- support for research data, including large and complex data types

- gold Open Access which fosters wider collaboration and increased citations

- maximum visibility for your research: over $100 \mathrm{M}$ website views per year

At BMC, research is always in progress.

Learn more biomedcentral.com/submissions 